\title{
Fluorescein and indocyanine-green angiography in ocular syphilis: an exploratory study
}

\author{
Konstantinos Balaskas • Theodoros N. Sergentanis • \\ Stefano Giulieri • Yan Guex-Crosier
}

Received: 14 June 2011 / Revised: 27 November 2011 / Accepted: 30 November 2011 / Published online: 25 December 2011

(C) Springer-Verlag 2011

\begin{abstract}
Background Fluorescein (FA) and indocyanine-green angiography (ICGA) may offer valuable information concerning disease severity and prognosis in ocular syphilis. The aim of the present study is to describe angiographic patterns encountered in the context of ocular syphilis, and to explore the associations between specific angiographic manifestations and severity of disease presentation, as well as disease evolution after treatment.

Methods We performed a retrospective institutional study with the inclusion of 23 patients with ocular syphilis presenting to the uveitis clinic of the Jules-Gonin Eye Hospital in a 10 -year period. FA and ICGA were performed following a standard protocol for posterior uveitis. Patterns of fluorescence were noted, and statistical associations between each angiographic pattern and any demographic, clinical, or laboratory parameter at baseline and after treatment were sought.

Results The presence of any dark dots in ICGA was significantly associated with anterior uveitis $(p=0.031)$. The
\end{abstract}

No financial support or funding was received in support of this study

K. Balaskas $\cdot$ Y. Guex-Crosier $(\bowtie)$

Jules-Gonin Eye Hospital, University of Lausanne,

Av. de France 15,

$\mathrm{CH}-1004$, Lausanne, Switzerland

e-mail: yan.guex@fa2.ch

T. N. Sergentanis

Department of Epidemiology and Biostatistics, Medical School,

University of Athens,

Athens, Greece

S. Giulieri

Department of Infectious Diseases,

Centre Hospitalier Universitaire Vaudois,

Lausanne, Switzerland presence of hot spots in ICGA was significantly associated with longer duration of symptoms prior to initial visit $(p=$ $0.032)$ and with male gender $(p=0.012)$. Weak nonsignificant trends were found associating vascular staining in FA with anterior uveitis $(p=0.066)$, vitritis $(p=0.069)$, and younger age $(p=0.061)$, as well as disc hyperfluorescence in FA with seropositivity for HIV $(p=0.089)$ and macular edema in FA with longer disease duration $(p=0.061)$. The presence of any dark dots in ICGA exhibited a weak trend of association with anterior uveitis and/or vitritis $(p=0.079)$. Conclusions Out of the several associations identified implicating specific angiographic features, we underline the possible role of the presence of dark dots in ICGA for identifying active inflammation, and the role of hot spots in ICGA as markers of long-standing disease. Vascular staining in FA appears to be more common in patients with severe ocular inflammation with presence of anterior uveitis and/or vitritis.

Keywords Ocular syphilis · Fluorescein angiography · Indocyanine-green angiogrpahy

\section{Introduction}

Ocular involvement in the context of syphilitic infection presents certain particularities stemming from the close anatomic proximity of the eye to the central nervous system. Ocular syphilis is therefore classified as a variant of neurosyphilis, irrespective of compatible findings in the cerebrospinal fluid. The classical description of the clinical course of syphilitic infection in three consecutive stages is less relevant in the presence of ocular involvement, as reflected in the Centers for Disease Control (CDC) guidelines for the management of syphilis that prompt clinicians to treat all 
cases of ocular syphilitic infection as neurosyphilis [1]. The value of laboratory investigations in determining disease severity and prognosis in ocular syphilis is limited. Although serum VDRL titers are considered appropriate for evaluating disease activity and response to treatment in syphilis, this is less clear in the context of ocular involvement, which is most common in the secondary, but also in the tertiary stage of the disease, when VDRL serology is almost invariably negative. Active and potentially debilitating ocular disease can therefore be accompanied by negative non-treponemal serology for syphilis [2]. Further laboratory investigations would therefore be needed to offer clues as regards disease severity and prognosis. The role of angiography in determining disease severity and in predicting disease evolution after treatment has not been adequately studied in ocular syphilis.

Fluorescein angiography (FA) has been extensively used for the evaluation of ocular inflammation in the context of posterior uveitis [3-5]. As fluorescein sodium fluoresces in the wavelengths of visible light, its value is mostly limited in revealing pathology of the superficial structures of the fundus [6]. Indocyanine-green (ICG), on the other hand, fluoresces on the infrared spectrum, giving access to the choroidal compartment through the retinal pigment epithelium. ICG leaks unimpaired from fenestrated choriocapillaris, albeit slowly, gradually impregnating choroidal tissue and giving rise to intermediate and late choroidal background fluorescence in the course of the angiogram. This fluorescence and its disturbance from choroidal inflammatory lesions are the main components studied in ICG angiography (ICGA) performed for posterior granulomatous uveitis [7-9].

FA manifestations of ocular syphilis have been reported in previous publications, identifying non-specific patterns of retinal inflammation, such as retinal and vascular staining, capillary leakage, macular edema, and optic disc hyperfluorescence [3-5]. A pattern unique to the disease has been described by Gass [10], corresponding to the entity named syphilitic posterior placoid chorioretinitis. ICGA patterns in ocular syphilis have been described in a case series by Mora et al., emphasizing the presence of late scattered hyperfluorescence as characteristic to the disease [11].

The purpose of the present study is to describe angiographic patterns in ocular syphilis both in fluorescein and ICG angiography, and to look for meaningful associations between specific angiographic findings and any baseline demographic or clinical parameter, as well as disease evolution after treatment and probability for relapse.

\section{Methods}

We retrospectively reviewed the charts of 26 patients with ocular inflammation and a positive treponemal serologic test for syphilis presenting to the Uveitis department of the Jules-Gonin Eye Hospital in the period between January 1999 and December 2009. Inclusion criteria for this study were signs of uveitis compatible with ocular syphilis on clinical evaluation, together with a positive TPHA or FTA-Abs test. Medical history and serology for each patient were discussed with an infectious disease specialist (SG), in order to ascertain diagnosis of syphilis and determine stage of disease progression. All patients were followed by the same uveitis specialist (YGC), and according to official recommendations laid down for the management of ocular syphilis [1]. Treatment consisted of either a standard regimen of intravenous penicillin for neurosyphilis at a dose of $6 \times 4 \mathrm{MO}$ IU per day for 14 days, or an alternative regimen of intravenous ceftriaxone at a dose of 2 gr per day for 14 days. Inclusion criteria, clinical and laboratory evaluations, and management are extensively discussed in a previous publication. Associations drawn from the same study sample as regards disease severity at baseline and disease evolution after treatment have also been previously reported [12]. Out of the 26 patients, three refused to enter into the proposed follow-up including fluorescein and ICG angiography, lumbar puncture, and visual field assessment. Twenty-three patients underwent dual fluorescein and ICG angiography following a standard protocol for the evaluation of posterior uveitis at the moment of disease diagnosis [13]. The same angiographic evaluation was performed after the completion of a treatment cycle for ocular syphilis in 17 patients.

As angiography constitutes an examination not readily quantifiable in clinical practice, and its interpretation remains to a large extent descriptive, we employed a qualitative approach to the recording of angiographic findings, noting the presence or absence of each characteristic pattern, but not its extent. This was mainly done in order to proceed to a concise, parsimonious statistical analysis of angiographic findings, given the small study sample.

Recorded angiographic patterns in FA included macular edema, disc hyperfluorescence, retinal staining, vascular staining, retinal ischemia, pinpoint leakage, mask effect from preretinal hemorrhage, window defects, and fluorescein leakage from choroidal or retinal neovascularization.

Recorded angiographic patterns in ICGA included: (1) persistent dark dots, defined as diversely sized hypofluorescent areas already present in the early and intermediate phases of the angiogram and remaining hypofluorescent in the late phase, (2) vanishing dark dots, different in that they became isofluorescent in the late phase of the angiogram, and (3) atrophic dark dots, a sub-variant of persistent dark dots remaining unaltered in subsequent angiograms after proper antibiotic treatment. Other noted angiographic features included hot spots, defined as scattered hyperfluorescent foci 
in the posterior pole or the mid-periphery in late angiographic frames, and fuzzy choroidal vessels observed in the intermediate phase of the angiogram.

With regard to statistical analysis of observed angiographic patterns, the following angiographic features were set as main factors in a series of univariate comparisons: retinal staining, vascular staining, disc hyperfluorescence, and macular edema in FA, as well as the presence of any dark dots and hot spots in ICGA. More specifically, the associations between the main factors and all registered demographic, clinical or laboratory parameters (ten in total) at baseline were thoroughly assessed. The aforementioned parameters included gender, age at diagnosis, unilateral or bilateral involvement, HIV status, duration of symptoms prior to initial visit, pain as a calling sign, anterior uveitis, vitritis, optic nerve involvement [as evidenced by a relative afferent pupillary defect (RAPD), and/or optic disc swelling], and relapse of inflammation after treatment. A further parameter, constituting an indicator of active ocular inflammation, was examined against all registered angiographic manifestations; the presence of anterior uveitis and/or vitritis vs the absence of both.

Inter-eye correlations implicating specific angiographic manifestations in cases with bilateral disease involvement were calculated in order to identify the degree of symmetry in FA and ICGA of ocular syphilis with bilateral involvement. Associations among the various angiographic manifestations both in FA and in ICGA were also sought.

Pearson's chi-square, Fisher's exact test and the MannWhitney-Wilcoxon test for independent samples (MWW) were appropriately applied in the light of deviation from normality (as evidenced from Kolmogorov-Smirnov and Shapiro-Wilk tests). With regard to continuous variables, median values and inter-quartile ranges are reported given the nonparametric statistical approach adopted. The level of statistical significance was set to $5 \%$. A patient-based analysis was performed in order to circumvent the potential inter-eye correlation in cases with bilateral involvement; the presence of any specific angiographic manifestation in any particular patient was only included once in the analysis irrespective of unilateral or bilateral involvement, thus eliminating the effect of inter-eye correlation. In simple terms, at the patient-based analysis, each patient was deemed positive regarding an angiographic manifestation when one or both eyes had exhibited the respective manifestation. Nevertheless, an alternative eye-based analysis was also performed, and is thoroughly presented in the Appendix, ensuring a comprehensive approach to available data. Given the exploratory nature of the present study, no adjustment of the significance level to account for multiple statistical comparisons was deemed to be warranted. Taking into account the small sample size, no multivariate analysis was performed. Observed associations may therefore be prone to mutual confounding which cannot be further investigated by multivariate analysis in the context of this study. Statistical analysis was performed with STATA 8.0 statistical software (Stata Corporation, College Station, TX, USA).

Institutional Review Board (IRB)/ Ethics Committee approval of the University of Lausanne was obtained. Described research adhered to the tenets of the Declaration of Helsinki.

\section{Results}

Simultaneous fluorescein and ICG angiography were performed in 23 patients ( 39 eyes) at presentation, following a standard angiographic protocol for the evaluation of posterior uveitis. The most frequent patterns of fluorescence in FA and ICGA are presented in Table 1. The features most frequently observed in FA were retinal staining of focal punctate retinal lesions $(43.5 \%)$, followed by staining of retinal vessels, mainly veins $(30.4 \%)$ and disc hyperfluorescence (30.4\%) (Fig. 1). Four patients presented a particular angiographic pattern consisting of hypofluorescent foci corresponding to the presence of chorioretinal scars, surrounded by hyperfluorescent areas in the late phase, an aspect compatible with syphilitic posterior placoid chorioretinitis. ICGA features could be classified into three main patterns. The first pattern consisted of hypofluorescent choroidal lesions, visible from the early and intermediate phases of the angiogram onwards, which either became isofluorescent in the late phases, or remained hypofluorescent. These lesions, also referred to as dark dots, varied considerably in size, number and localisation, sharing the common characteristic of being invisible in fluorescein angiography or

Table 1 Findings in fluorescein and indocyanine-green angiography ( $n=23$ and 22 patients respectively)

$N(\%)$

Findings in fluoroangiography $(n=23)$

Disc hyperfluorescence

7 (30.4)

Macular edema

4 (17.4)

Vascular staining

7 (30.4)

Retinal staining and/or capillary leakage

$10(43.5)$

Retinal ischemia

$2(8.7)$

Normal

$2(8.7)$

Findings in ICGA $(n=22)$

Dark dots

Persistent dark dots exclusively

Persistent and vanishing dark dots

$10(45.5)$

Hot spots

Fuzzy choroidal vessels

$5(14.7)$

Normal

$3(8.8)$ 
Fig. 1 a Fundus photograph depicting snowballs in the vitreous. b, d Extensive central and peripheral retinal staining and/or capillary leakage in FA. c Foci of peripheral retinal vascular staining in FA
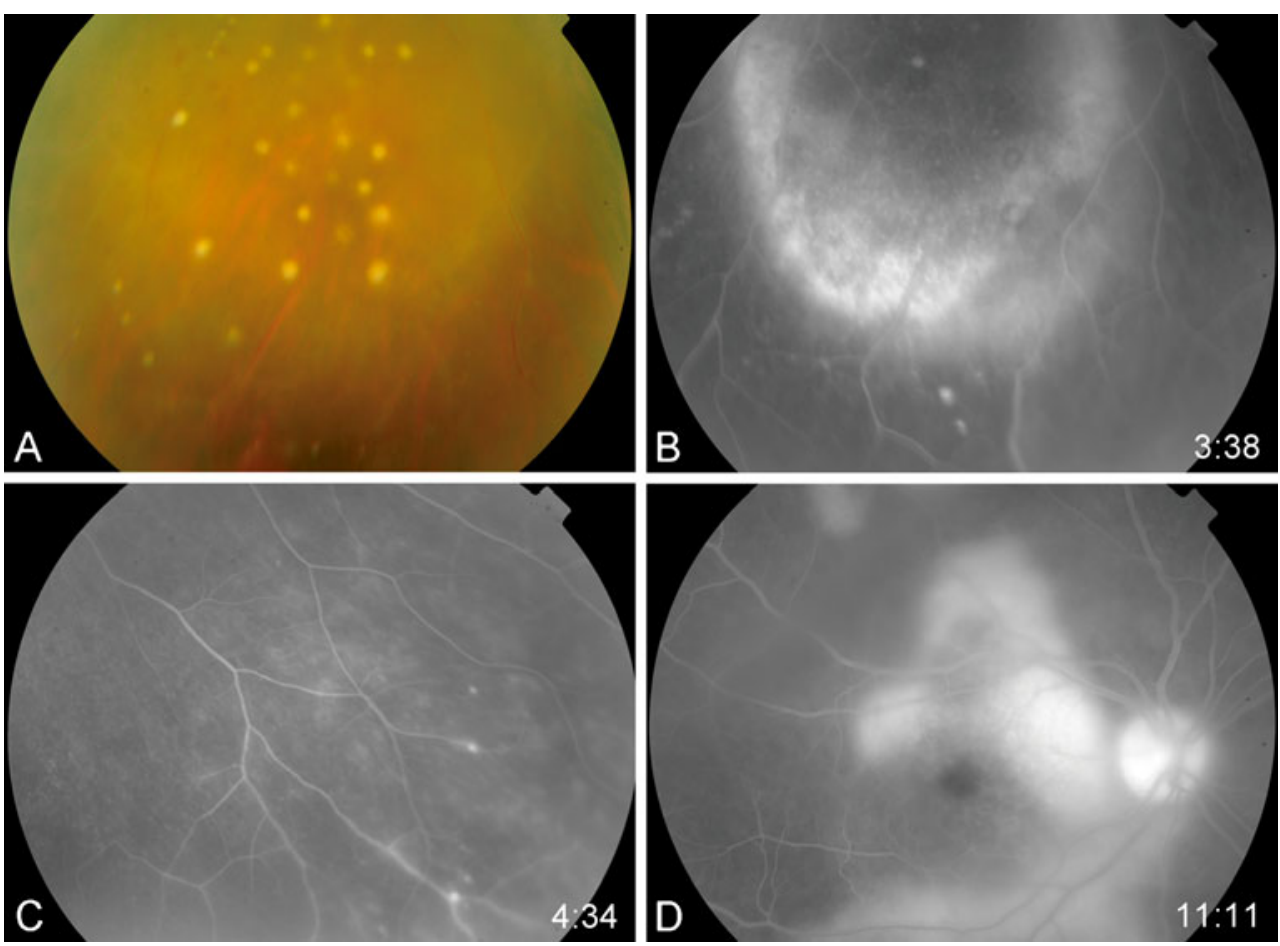

fundoscopy, and were observed in 13 patients (59.1\%) (Fig. 2). The second pattern corresponded to scattered hyperfluorescent spots (hot spots) appearing in the late phases of the angiogram, in the mid-periphery and/or the posterior pole present in 11 patients $(50 \%)$. The third pattern was fuzzy choroidal vessels with leakage from the intermediate phase of the angiogram onwards in five patients $(14.7 \%)$. In $13.6 \%$ of patients, the persistent variant of dark dots that remained unaltered in the late frames of the angiogram was observed, while $45.5 \%$ of patients presented both persistent and vanishing dark dots, the latter becoming isofluorescent in late frames. Seventeen patients were submitted to the same angiographic investigations after the completion of an antibiotic treatment regimen for neurosyphilis. Where comparison was possible, in FA vascular staining disappeared after treatment in five out of seven cases (71.4\%), disc hyperfluorescence in five out of seven cases (71.4\%), and retinal staining in four out of ten cases (40\%). In ICGA, three cases of persistent dark dots remaining unaltered in size after treatment, corresponding to chorioretinal scars, were identified. Hot spots disappeared in almost every case after treatment ( 9 out of 11 patients, $82 \%$ ) (Fig. 3).

Out of the 23 patients, 13 had inflammatory signs evidenced by the presence of anterior uveitis and/or vitritis, whereas ten did not. Out of the latter patients, six had concomitant optic nerve involvement, two had posterior placoid chorioretinitis, three had retinal staining in FA, and only one had vascular staining, whereas no patient without anterior uveitis and/or vitritis presented with macular edema. With regard to dark dots, which were more common in eyes with anterior uveitis and/or vitritis though not in a statistically significant way, they were present in all four cases that presented relapse of inflammation after proper antibiotic treatment.
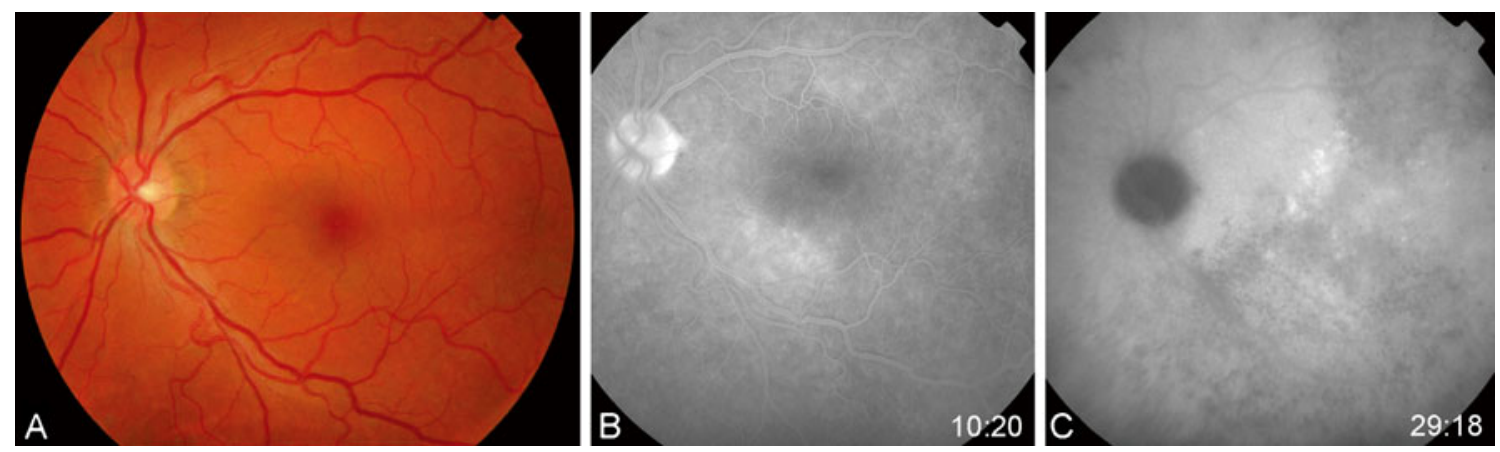

Fig. 2 a Fundus photograph depicting normal retina. b FA of the same case revealing minimal retinal staining. $\mathbf{c}$ ICGA of the same case revealing extensive dark dots and some hot spots, invisible on both fundus photograph and FA 
Fig. 3 a ICGA revealing hot spots. b Disappearance of hot spots after treatment
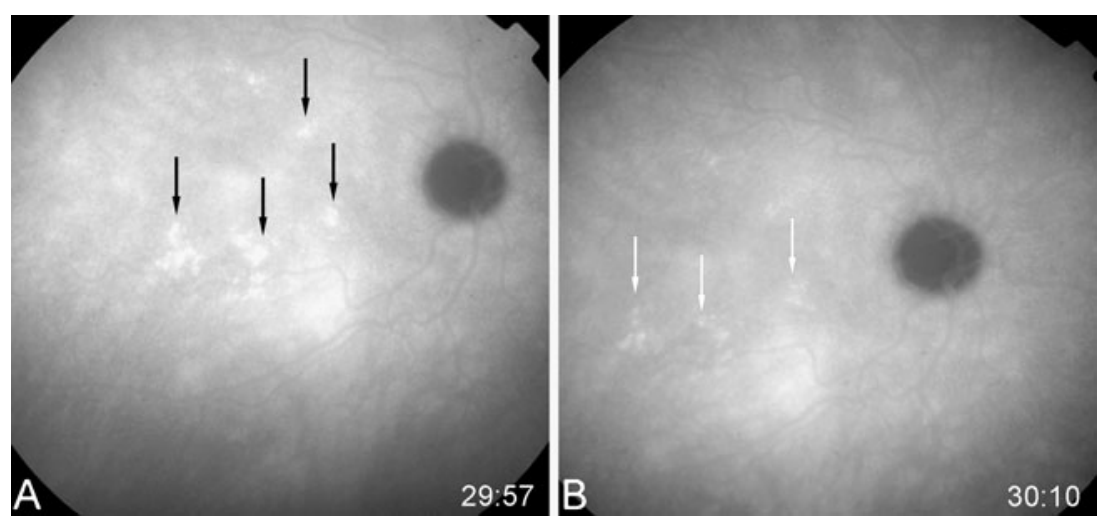

Associations among the various angiographic manifestations in FA and ICGA are presented in the triangular Table 2. It is obvious that no significant associations could be identified, other than a weak trend implicating vascular staining and macular edema in FA ( $p=0.067$, Fisher's exact test), though not reaching statistical significance.

Associations between the two most frequent angiographic manifestations in FA, retinal staining and vascular staining, and all registered baseline factors are presented in Tables 3 and 4 respectively. With regard to associations implicating specific angiographic features, vascular staining in FA exhibited a weak trend of association with vitritis (6/ 12 patients with vitritis also exhibited vascular staining in FA, whereas only $1 / 11$ patients without vitritis exhibited vascular staining, $p=0.069$, Fisher's exact test), anterior uveitis (5/9 patients with anterior uveitis presented with vascular staining vs $2 / 14$ patients without anterior uveitis, $p=0.066$, Fisher's exact test) and younger age at presentation [39.0 years (inter-quartile range (IQR) 9.2] for patients with vascular staining vs 47.8 (IQR 14.8) for those without, $p=0.061$, Mann-Whitney-Wilcoxon test), though none of the associations reached statistical significance. A strong association between presence of anterior uveitis and presence of vitritis was also identified ( $p=0.015$, Fisher's exact test). The association between vascular staining in fluorescein angiography with worse visual acuity at baseline did not reach formal statistical significance $(p=0.072, \mathrm{MWW})$, though there was a significant association with improvement in visual acuity after treatment $(p=0.005)$, as exhaustively described in a previous publication [12]. There was a weak trend of association between disc hyperfluorescence in FA and seropositivity for HIV infection (2/2 patients with HIV also exhibited disc hyperfluorescence vs only $5 / 21$ patients without HIV infection, $p=0.083$, Fisher's exact test) as well as between macular edema in FA and longer disease duration prior to initial presentation [225 days (IQR 122) for patients with macular edema vs 12 (IQR 23) for patients without macular edema, $p=$ 0.061, MWW], though the associations did not reach statistical significance. As expected, disc hyperfluorescence was significantly associated with optic nerve involvement $(p<$ 0.001). All other associations implicating specific FA manifestations were non-significant.

Associations between the presence of any dark dots in ICGA and any other parameter are presented in Table 5. The presence of any dark dots was significantly associated with anterior uveitis ( $p=0.031$, Fisher's), while dark dots were more common in patients with anterior uveitis and/or vitritis ( $p=0.079$, Fisher's). The presence of any hot spots in ICGA was associated with male gender ( $p=0.012$, Fisher's) and longer duration of symptoms prior to initial visit $(p=0.032$, MWW), as can be seen in Table 6. The former association remained significant even after the exclusion of patients positive for HIV infection ( $p=0.008$, Fisher's). There was, however, no association between hot spots and any sign of active ocular inflammation.

In cases with bilateral disease, angiographic manifestations strongly correlated between fellow eyes as follows: retinal staining ( $\mathrm{rho}=0.854, p=0.005)$, vascular staining

Table 2 Associations between the various angiographic manifestations in FA and ICGA

\begin{tabular}{|c|c|c|c|c|c|c|}
\hline & Retinal staining & Vascular staining & Disc hyperfluorescence & Macular edema & Dark dots & Hot spots \\
\hline Retinal staining & - & & & & & \\
\hline Vascular staining & 0.405 & - & & & & \\
\hline Disc hyperfluorescence & 0.405 & $>0.999$ & 一 & & & \\
\hline Macular edema & 0.104 & 0.067 & 0.557 & - & & \\
\hline Dark dots & 0.674 & 0.648 & 0.648 & 0.616 & - & \\
\hline Hot spots & $>0.999$ & $>0.999$ & $>0.999$ & $>0.999$ & $>0.999$ & - \\
\hline
\end{tabular}


Table 3 Variables associated with retinal staining (patient-based analysis). Percentages are given with $100 \%$ corresponding to each row

\begin{tabular}{|c|c|c|c|}
\hline & $\begin{array}{l}\text { Retinal } \\
\text { staining }\end{array}$ & $\begin{array}{l}\text { No retinal } \\
\text { staining }\end{array}$ & \\
\hline Categorical variables & Freq $(\%)$ & Freq $(\%)$ & $P$ \\
\hline Gender & & & $>0.999^{\mathrm{F}}$ \\
\hline Male $(n=16)$ & $7(43.8)$ & $9(56.3)$ & \\
\hline Female $(n=7)$ & $3(42.9)$ & $4(57.1)$ & \\
\hline Involvement & & & $0.402^{\mathrm{F}}$ \\
\hline Unilateral $(n=10)$ & $3(30.0)$ & $7(70.0)$ & \\
\hline Bilateral $(n=13)$ & $7(53.9)$ & $6(46.2)$ & \\
\hline HIV status & & & $0.486^{\mathrm{F}}$ \\
\hline Positive $(n=2)$ & $0(0.0)$ & $2(100.0)$ & \\
\hline Negative $(n=21)$ & $10(47.6)$ & $11(52.4)$ & \\
\hline Pain as a calling sign & & & $0.074^{\mathrm{F}}$ \\
\hline Yes $(n=8)$ & $1(12.5)$ & $7(87.5)$ & \\
\hline No $(n=15)$ & $9(60.0)$ & $6(40.0)$ & \\
\hline Optic neuropathy & & & $>0.999^{\mathrm{F}}$ \\
\hline Yes $(n=9)$ & $4(44.4)$ & $5(55.6)$ & \\
\hline No $(n=14)$ & $6(42.9)$ & $8(57.1)$ & \\
\hline Anterior uveitis & & & $>0.999^{\mathrm{F}}$ \\
\hline Yes $(n=9)$ & $4(44.4)$ & $5(55.6)$ & \\
\hline No $(n=14)$ & $6(42.9)$ & $8(57.1)$ & \\
\hline Vitritis & & & $0.214^{\mathrm{F}}$ \\
\hline Yes $(n=12)$ & $7(58.3)$ & $5(41.7)$ & \\
\hline No $(n=11)$ & $3(27.3)$ & $8(72.7)$ & \\
\hline $\begin{array}{l}\text { Anterior uveitis } \\
\text { and/or vitritis** }\end{array}$ & & & $0.402^{\mathrm{F}}$ \\
\hline Yes $(n=13)$ & $7(53.9)$ & $6(46.1)$ & \\
\hline No $(n=10)$ & $3(30.0)$ & $6(70.0)$ & \\
\hline Relapse after treatment & & & $0.582^{\mathrm{F}}$ \\
\hline Yes $(n=4)$ & $1(25.0)$ & $3(75.0)$ & \\
\hline No $(n=15)$ & $8(53.3)$ & $7(46.7)$ & \\
\hline Continuous variables & $\begin{array}{r}\text { Median } \\
(\mathrm{IQR})\end{array}$ & $\begin{array}{r}\text { Median } \\
(\mathrm{IQR})\end{array}$ & $P$ \\
\hline Age (years) & $43.0(11.5)$ & $47.6(11.8)$ & $0.385^{\mathrm{MWW}}$ \\
\hline $\begin{array}{l}\text { Duration of symptoms } \\
\text { prior to initial visit } \\
\text { (days) }\end{array}$ & $36(87)$ & $90(146)$ & $0.755^{\mathrm{MWW}}$ \\
\hline
\end{tabular}

F Fisher's exact test

MwW Mann-Whitney-Wilcoxon test

$($ rho $=0.779, p=0.038)$, macular edema ( $\mathrm{rho}=0.779, p=$ $0.038)$, dark dots $(\mathrm{rho}=0.810, p=0.024)$, and hot spots $($ rho $=0.837, p=0.010)$. Disc hyperfluorescence did not exhibit a significant correlation in cases with bilateral disease (rho=0.426, $p=0.203$ ), with rho values corresponding to Pearson's correlation coefficient and $p$-values deriving from Fisher's exact test. In the eye-based analysis, a shift was observed of some statistically non-significant results towards statistical significance, as is thoroughly presented in the Appendix.
Table 4 Variables associated with vascular staining (patient-based analysis). Percentages are given with $100 \%$ corresponding to each row

\begin{tabular}{lll}
\hline $\begin{array}{l}\text { Vascular } \\
\text { staining }\end{array}$ & $\begin{array}{l}\text { No vascular } \\
\text { staining }\end{array}$ \\
\hline
\end{tabular}

Categorical variables $\quad$ Freq (\%) $\quad$ Freq (\%) $\quad P$

\section{Gender}

Male $(n=16)$

Female $(n=7)$

Involvement

Unilateral $(n=10)$

$5(31.2)$

$11(68.8)$

2 (28.6) $5(71.4)$

Bilateral $(n=13)$

HIV status

Positive $(n=2)$

Negative $(n=21)$

Pain as a calling sign

Yes $(n=8)$

No $(n=15)$

Optic neuropathy

Yes $(n=9)$

No $(n=14)$

Anterior uveitis

Yes $(n=9)$

No $(n=14)$

Vitritis

Yes $(n=12)$

No $(n=11)$

Anterior uveitis and/or vitritis**

Yes $(n=13)$

No $(n=10)$

$4(40.0)$

$6(60.0)$

$3(23.1) \quad 10(76.9)$

$1(50.0) \quad 1(50.0)$

6 (28.6) $\quad 15(71.4)$

$4(50.0) \quad 4(50.0)$

$3(20.0) \quad 12(80.0)$

$3(33.3) \quad 6(66.7)$

4 (28.6) $\quad 10(71.4)$

$5(55.6) \quad 4(44.4)$

$2(14.3) \quad 12(85.7)$

$6(50.0) \quad 6(50.0)$

$1(9.1) \quad 10(90.9)$

Relapse after treatment

Yes $(n=4)$

No $(n=15)$

Continuous variables

Age (years)

Duration of symptoms prior to initial visit (days)

\footnotetext{
${ }^{\mathrm{F}}$ Fisher's exact test

MwW Mann-Whitney-Wilcoxon test
}

\section{Discussion}

We conducted this exploratory study in search of associations between specific angiographic manifestations and several baseline demographic, clinical, or laboratory parameters. As regards the associations implicating specific angiographic manifestations in FA, only the one between vascular staining and improvement in visual acuity after treatment reached formal statistical significance. Several weak trends of association were, however, highlighted in this analysis, specifically 
Table 5 Variables associated with dark dots (patient-based analysis). Percentages are given with $100 \%$ corresponding to each row

\begin{tabular}{|c|c|c|c|}
\hline & Dark dots & $\begin{array}{l}\text { No dark } \\
\text { dots }\end{array}$ & \\
\hline Categorical variables & Freq $(\%)$ & Freq $(\%)$ & $P$ \\
\hline Gender & & & $>0.999^{\mathrm{F}}$ \\
\hline Male $(n=16)$ & $9(56.3)$ & $7(43.7)$ & \\
\hline Female $(n=6)$ & $4(66.7)$ & $2(33.3)$ & \\
\hline Involvement & & & $0.192^{\mathrm{F}}$ \\
\hline Unilateral $(n=10)$ & $4(40.0)$ & $6(60.0)$ & \\
\hline Bilateral $(n=12)$ & $9(75.0)$ & $3(25.0)$ & \\
\hline HIV status & & & $>0.999^{\mathrm{F}}$ \\
\hline Positive $(n=2)$ & $1(50.0)$ & $1(50.0)$ & \\
\hline Negative $(n=20)$ & $12(60.0)$ & $8(40.0)$ & \\
\hline Pain as a calling sign & & & $>0.999^{\mathrm{F}}$ \\
\hline Yes $(n=8)$ & $5(62.5)$ & $3(37.5)$ & \\
\hline No $(n=14)$ & $8(57.1)$ & $6(42.9)$ & \\
\hline Optic neuropathy & & & $0.674^{\mathrm{F}}$ \\
\hline Yes $(n=9)$ & $6(66.7)$ & $3(33.3)$ & \\
\hline No $(n=13)$ & $7(53.9)$ & $6(46.1)$ & \\
\hline Anterior uveitis & & & $0.031^{\mathrm{F}}$ \\
\hline Yes $(n=9)$ & $8(88.9)$ & $1(11.1)$ & \\
\hline No $(n=13)$ & $5(38.5)$ & $8(61.5)$ & \\
\hline Vitritis & & & $0.192^{\mathrm{F}}$ \\
\hline Yes $(n=12)$ & $9(75.0)$ & $3(25.0)$ & \\
\hline No $(n=10)$ & $4(40.0)$ & $6(60.0)$ & \\
\hline $\begin{array}{l}\text { Anterior uveitis } \\
\text { and/or vitritis** }\end{array}$ & & & $0.079^{\mathrm{F}}$ \\
\hline Yes $(n=13)$ & $10(76.9)$ & $3(23.1)$ & \\
\hline No $(n=9)$ & $3(33.3)$ & $6(66.7)$ & \\
\hline Relapse after treatment & & & $0.245^{\mathrm{F}}$ \\
\hline Yes $(n=4)$ & $4(100.0)$ & $0(0.0)$ & \\
\hline No $(n=15)$ & $8(53.3)$ & $7(46.7)$ & \\
\hline Continuous variables & $\begin{array}{l}\text { Median } \\
\text { (IQR) }\end{array}$ & $\begin{array}{r}\text { Median } \\
(\mathrm{IQR})\end{array}$ & $P$ \\
\hline Age (years) & $45.1(9.1)$ & $42.2(11.8)$ & $0.867^{\mathrm{MWW}}$ \\
\hline $\begin{array}{l}\text { Duration of symptoms } \\
\text { prior to initial visit } \\
\text { (days) }\end{array}$ & $12(147)$ & $90(83)$ & $0.867^{\mathrm{MWW}}$ \\
\hline
\end{tabular}

F Fisher's exact test

Mww Mann-Whitney-Wilcoxon test

between vascular staining and anterior uveitis, vitritis, anterior uveitis and/or vitritis, worse visual acuity at presentation, and younger age at presentation, as well as between disc hyperfluorescence and seropositivity for HIV and between macular edema and longer disease duration prior to the initial visit. The findings implicating vascular staining may point to a close association between these markers of active inflammation, indicating that eyes with vascular staining are more severely inflamed, which in turn may account for the weak trend towards worse BCVA at baseline in these patients. As
Table 6 Variables associated with hot spots (patient-based analysis). Percentages are given with $100 \%$ corresponding to each row

\begin{tabular}{|c|c|c|c|}
\hline & Hot Spots & $\begin{array}{l}\text { No Hot } \\
\text { Spots }\end{array}$ & \\
\hline Categorical variables & Freq( $(\%)$ & Freq $(\%)$ & $\mathrm{p}$ \\
\hline Gender* & & & $0.012^{\mathrm{F}}$ \\
\hline Male $(n=16)$ & $11(68.8)$ & $5(31.2)$ & \\
\hline Female $(n=6)$ & $0(0.0)$ & $6(100.0)$ & \\
\hline Involvement & & & $0.670^{\mathrm{F}}$ \\
\hline Unilateral $(n=10)$ & $6(60.0)$ & $4(40.0)$ & \\
\hline Bilateral $(n=12)$ & $5(41.7)$ & $7(58.3)$ & \\
\hline HIV status & & & $0.476^{\mathrm{F}}$ \\
\hline Positive $(n=2)$ & $0(0.0)$ & $2(100.0)$ & \\
\hline Negative $(n=20)$ & $11(55.0)$ & $9(45.0)$ & \\
\hline Pain as a calling sign & & & $0.659^{\mathrm{F}}$ \\
\hline Yes $(n=8)$ & $3(37.5)$ & $5(62.5)$ & \\
\hline No $(n=14)$ & $8(57.1)$ & $6(42.9)$ & \\
\hline Optic neuropathy & & & $>0.999^{\mathrm{F}}$ \\
\hline Yes $(n=9)$ & $4(44.4)$ & $5(55.6)$ & \\
\hline No $(n=13)$ & $7(53.9)$ & $6(46.1)$ & \\
\hline Anterior uveitis & & & $>0.999^{\mathrm{F}}$ \\
\hline Yes $(n=9)$ & $4(44.4)$ & $5(55.6)$ & \\
\hline No $(n=13)$ & $7(53.9)$ & $6(46.1)$ & \\
\hline Vitritis & & & $0.670^{\mathrm{F}}$ \\
\hline Yes $(n=12)$ & $7(58.3)$ & $5(41.7)$ & \\
\hline No $(n=10)$ & $4(40.0)$ & $6(60.0)$ & \\
\hline $\begin{array}{l}\text { Anterior uveitis } \\
\text { and/or vitritis** }\end{array}$ & & & $0.387^{\mathrm{F}}$ \\
\hline Yes $(n=13)$ & $8(61.5)$ & $5(38.5)$ & \\
\hline No $(n=9)$ & $3(33.3)$ & $6(66.7)$ & \\
\hline Relapse after treatment & & & $0.582^{\mathrm{F}}$ \\
\hline Yes $(n=4)$ & $1(25.0)$ & $3(75.0)$ & \\
\hline No $(n=15)$ & $8(53.3)$ & $7(46.7)$ & \\
\hline Continuous variables & $\begin{array}{r}\text { Median } \\
(\mathrm{IQR})\end{array}$ & $\begin{array}{l}\text { Median } \\
\text { (IQR) }\end{array}$ & $P$ \\
\hline Age (years) & $39.0(18.4)$ & $45.1(8.9)$ & $0.376^{\mathrm{MWW}}$ \\
\hline $\begin{array}{l}\text { Duration of symptoms } \\
\text { prior to initial visit } \\
\text { (days) }\end{array}$ & $90(210)$ & $7(59)$ & $0.032^{\mathrm{MWW}}$ \\
\hline
\end{tabular}

* After the exclusion of patients with HIV infection, the association persists $(11 / 15$ males vs $0 / 5$ females exhibit hot spots in ICG, $p=0.008)$

F Fisher's exact test

Mww Mann-Whitney-Wilcoxon test

evidenced in our previous study, improvement in visual acuity after treatment was significantly associated with presence of vascular staining in FA, underlining the reversible nature of this type of ocular inflammatory reaction in the context of ocular syphilis [12]. The high rate of disappearance of vascular staining in FA performed after treatment points to the same conclusion, indicating that vasculitis is not accompanied by permanent visual repercussions in ocular syphilis, when properly treated. The fact that all patients with HIV seropositivity 
exhibited disc hyperfluorescence in FA, illustrates a probable previously unrecognised site of preferential posterior involvement of ocular syphilis in the context of HIV co-infection. The potential increased prevalence of macular edema in patients with longer duration of intra-ocular inflammation has been suspected in uveitis [14], and may point to a deleterious effect of persistent inflammatory stimuli, leading to gradual accumulation of intraretinal fluid in the macular area. The aspect of "leopard spots" in fluorescein angiography described by Gass [10] in the context of syphilitic posterior placoid chorioretinitis as being highly indicative of syphilitic infection was also observed in four patients in our series. Interestingly, patients without anterior uveitis and/or vitritis mainly presented with optic nerve involvement, and to a lesser extent posterior placoid chorioretinitis and retinal staining, though only one such patient exhibited vascular staining, and none had macular edema.

Similar patterns in ICGA to those observed in our series have been described in other forms of granulomatous posterior uveitis, such as sarcoidosis and tuberculosis $[15,16]$, and are not unique to ocular syphilis. Probable interpretations for the above mentioned patterns are atrophy, fibrosis, or full-thickness granuloma for persistent dark dots, partial thickness granuloma or delayed perfusion of choriocapillaris for vanishing dark dots, and choroidal vasculitis for fuzzy choroidal vessels. Dark dots have also been described in ocular sarcoidosis and ocular tuberculosis, the latter allegedly being associated with more extensive and more confluent lesions [17]. Our study revealed a significant association between presence of dark dots and anterior uveitis, and a weak trend of association with presence of anterior uveitis and/or vitritis. It should be emphasized that there was a strong association between the presence of anterior uveitis and the presence of vitritis, prompting us to introduce a combined parameter implicating both inflammatory indicators and to test all baseline factors against this parameter as well. Dark dots were present in all four patients that exhibited relapse of inflammation after initial remission following proper antibiotic treatment, though no statistically significant association could be drawn from such small numbers. We suggest that the presence of dark dots, excluding those associated with atrophy, may signify active granulomatous posterior choroidal inflammation. Due to the small sample, however, we were unable to identify the specific characteristics of dark dots, for instance size, number, and localisation, which are more evocative of active inflammation.

With regard to hot spots, although they have been observed in other granulomatous uveitis, their high prevalence in ocular syphilis could be characteristic to the disease. These features have been described in the article by Mora et al. [11], and probable proposed interpretations include fixing of ICG molecules on active granuloma or staining of leucocytes. These manifestations have also been alleged to indicate active disease or long-standing disease. The former assumption was not, however, statistically confirmed in the present study. On the contrary, the lack of association between hot spots and any ocular manifestation with important functional repercussions, such as macular edema, optic neuropathy, or anterior uveitis, may reveal an exclusively choroidal origin of these angiographic features. On the other hand, the identified association between the presence of hot spots and a longer duration of symptoms prior to the first visit may render this angiographic finding indicative of long-standing disease. Hot spots appeared to be significantly more common in male patients. This association, though persisting even after the exclusion of patients with HIV co-infection, was rather more difficult to interpret.

Surprisingly, no association could be identified between various angiographic manifestations both in FA and ICGA, other than a weak positive trend implicating vascular staining and macular edema. Although both vascular staining in FA and dark dots in ICGA appear to be more common in eyes with anterior uveitis and/or vitritis, they do not seem to coexist in the same eye. This observation, in conjunction with the absence of any other association between ICGA findings and any clinical parameter indicative of retinal inflammation such as retinal staining or macular edema, could justify the speculation of two morphologically and angiographically distinct patterns of syphilitic eye disease, namely a predominantly choroidal versus a predominantly retinal variant.

Inter-eye correlation of angiographic manifestations in case of bilateral disease involvement appears significant and strong as regards all main factors apart from disc hyperfluorescence. This analysis makes it possible to provide useful insight into the degree of correlation of angiographic findings between fellow eyes in bilateral ocular syphilis, which has not so far been reported. Given the high degree of inter-eye correlation of angiographic manifestations identified, the use of a patient-based analysis becomes even more appropriate in the context of the present study.

This study has certain limitations stemming mainly from its retrospective design and the small study sample. A further shortcoming lies in the loss of six patients to follow-up with regard to angiographic evaluation after treatment, further limiting our ability to assess the effect of treatment on observed angiographic patterns. It should also be emphasized that given the small study sample, multivariate analysis could not be performed so as to avoid over-interpretation of available data. Observed associations may therefore be prone to mutual confounding, and their independence may not be warranted.

We conclude, based on our findings, that angiographic investigation in ocular syphilis can offer valuable information concerning disease severity and duration, and can even contribute some clues to disease prognosis. Vascular staining in FA and dark dots in ICGA appear to be more common in more severely inflamed eyes with anterior uveitis and/or vitritis, whereas hot spots are more often present in patients with long-standing disease without other signs of severe 
inflammation. Two variants of the disease, one with predominantly choroidal involvement and one predominantly retinal, could be hypothesized. In view, however, of the exploratory nature of the current study and the above-mentioned limitations, further corroborative studies are required to confirm our findings. Nevertheless, some earlier arbitrary hypotheses concerning the significance of specific angiographic features in granulomatous posterior uveitis were for the first time statistically investigated in the present study.

Acknowledgments This article was the object of an oral presentation entitled "Angiographic patterns in ocular syphilis" at the EVER 2010 Congress held in Crete, Greece between 6 and 9 October 2010

We particularly thank Catherine Guex-Crosier for her valuable contribution to data collection and management.

\section{Appendix}

An eye-based analysis of registered data was performed in the service of comprehensiveness and in an attempt to exploit all available data. Angiographic manifestations encountered in FA and ICGA on an eye basis are presented in Table 7. With regard to associations implicating specific angiographic features, vascular staining in FA exhibited a statistically significant association with vitritis (8/19 eyes with vitritis also exhibited vascular staining in FA, whereas only $1 / 17$ eyes without vitritis exhibited vascular staining, $p=0.020)$, anterior uveitis (7/15 eyes with anterior uveitis presented with vascular staining vs $2 / 21$ eyes without anterior uveitis, $p=0.019$ ) and a weak trend implicating macular edema (4/8 eyes with macular oedema vs $5 / 28$ eyes without macular edema exhibited vascular staining, $p=0.086$ ), with $p$-values derived from Fisher's exact test. The association between

Table 7 Findings in fluorescein and indocyanine-green angiography $(n=39$ eyes $)$

\begin{tabular}{ll}
\hline & $N(\%)$ \\
\hline Findings in fluoroangiography $(n=36)$ & \\
Disc hyperfluorescence & $9(25.0)$ \\
Macular edema & $5(13.9)$ \\
Vascular staining & $9(25.0)$ \\
Retinal staining and/or capillary leakage & $15(41.7)$ \\
Retinal ischemia & $3(6.5)$ \\
Normal & $3(6.5)$ \\
Findings in ICGA ( $n=34)$ & \\
Dark dots & $21(61.8)$ \\
Persistent dark dots & $4(11.8)$ \\
Persistent and vanishing dark dots & $17(50.0)$ \\
Hot spots & $15(44.1)$ \\
Fuzzy choroidal vessels & $5(14.7)$ \\
Normal & $3(8.8)$ \\
\hline
\end{tabular}

Table 8 Variables associated with dark dots (eye-based analysis). Percentages are given with $100 \%$ corresponding to each row

\begin{tabular}{llll}
\hline & Dark dots & No dark dots & \\
\hline $\begin{array}{l}\text { Categorical variables } \\
\text { Anterior uveitis }\end{array}$ & Freq(\%) & Freq(\%) & $P$ \\
Yes $(n=15)$ & $13(86.7)$ & $2(13.3)$ & $0.013^{\mathrm{F}}$ \\
No $(n=19)$ & $8(42.1)$ & $11(57.9)$ & \\
Vitritis & & & $0.034^{\mathrm{F}}$ \\
Yes $(n=19)$ & $15(79.0)$ & $4(21.0)$ & \\
No $(n=15)$ & $6(40.0)$ & $9(60.0)$ & $0.427^{\mathrm{F}}$ \\
Vascular staining in FA & & & \\
Yes $(n=9)$ & $7(77.8)$ & $2(22.2)$ & $0.066^{\mathrm{F}}$ \\
No $(n=25)$ & $14(56.0)$ & $11(44.0)$ & \\
Relapse after treatment & & & \\
Yes $(n=7)$ & $7(100.0)$ & $0(0.0)$ & $10(41.7)$ \\
No $(n=24)$ & $14(58.3)$ & & \\
\hline F $p$-value derived from Fisher's exact test &
\end{tabular}

vascular staining in fluorescein angiography with worse visual acuity at baseline did not reach formal statistical significance $(p=0.072, \mathrm{MWW})$, though there was a significant association with improvement in visual acuity after treatment $(p=0.005)$, as exhaustively described in a previous publication [12]. Disc hyperfluorescence in FA was significantly associated with seropositivity for HIV infection ( $3 / 3$ eyes with HIV also exhibited disc hyperfluorescence vs only $6 / 33$ eyes without HIV infection, $p=0.012$, Fisher's exact test).

Associations between the presence of any dark dots in ICGA and any other parameter are presented in Table 8. The presence of any dark dots was significantly associated with

Table 9 Variables associated with hot spots (eye-based analysis). Percentages are given with $100 \%$ corresponding to each row

\begin{tabular}{|c|c|c|c|}
\hline & Hot spots & $\begin{array}{l}\text { No hot } \\
\text { spots }\end{array}$ & \\
\hline Categorical variables & Freq $(\%)$ & Freq $(\%)$ & $\mathrm{P}$ \\
\hline Gender & & & $<0.001^{\mathrm{F}}$ \\
\hline Male $(n=23)$ & $15(65.2)$ & $8(34.8)$ & \\
\hline Female $(n=11)$ & $0(0.0)$ & $11(100.0)$ & \\
\hline Relapse & & & $0.201^{\mathrm{F}}$ \\
\hline Yes $(n=7)$ & $1(14.3)$ & $6(85.7)$ & \\
\hline No $(n=24)$ & $11(45.8)$ & $13(54.2)$ & \\
\hline Continuous variables & $\begin{array}{r}\text { Median } \\
(\mathrm{IQR})\end{array}$ & $\begin{array}{r}\text { Median } \\
(\mathrm{IQR})\end{array}$ & $P$ \\
\hline $\begin{array}{l}\text { Duration of symptoms } \\
\text { prior to initial visit } \\
\text { (days) }\end{array}$ & 7 (149) & $120(210)$ & $0.007^{\mathrm{MWW}}$ \\
\hline
\end{tabular}


anterior uveitis $(p=0.013)$, vitritis $(p=0.034)$, while dark dots were observed in all seven eyes with relapse of inflammation after proper antibiotic treatment $(p=0.066)$. The presence of any hot spots in ICGA was associated with male gender $(p<0.001)$ and longer duration of symptoms prior to initial visit $(p=0.007)$, as can be seen in Table 9. There was, however, no association with relapse of inflammation after treatment $(p=0.201)$.

\section{References}

1. Workowski KA, Berman S (2006) Sexually transmitted disease treatment guidelines. MMWR Morb Mortal Wkly Rep 55:22-26

2. Browning DJ (2000) Posterior segment manifestations of active ocular syphilis, their response to a neurosyphilis regimen of penicillin therapy, and the influence of human immunodeficiency virus status on response. Ophthalmology 107:2015-2023

3. Sudharshan S, Ganesh SK, Biswas J (2010) Current approach in the diagnosis and management of posterior uveitis. Indian J Ophthalmol 58:29-43

4. Tan-Yaycioglu R, Akova YA, Akca S, Yilmaz G (2006) Inflammation of the posterior uvea: findings on fundus fluorescein and indocyanine green angiography. Ocul Immunol Inflamm 14:171-179

5. De Laey JJ (1995) Fluorescein angiography in posterior uveitis. Int Ophthalmol Clin 35:33-58

6. Patz A (1977) Principles of fluorescein angiography. Int Ophthalmol Clin 17:1-19
7. Herbort CP, Bodaghi B, Lehoang P (2001) Indocyanine green angiography in ocular inflammatory diseases: principles, schematic interpretation, semiology and clinical value. J Fr Ophtalmol 24:423-447

8. Desmettre T, Devoisselle JM, Mordon S (2000) Fluorescence properties and metabolic features of indocyanine green (ICG) as related to angiography. Surv Ophthalmol 45:15-27

9. Mordon S, Devoisselle JM, Soulie-Begu S, Desmettre T (1998) Indocyanine green: physicochemical factors affecting its fluorescence in vivo. Microvasc Res 55:146-152

10. Gass JD, Braunstein RA, Chenoweth RG (1990) Acute syphilitic posterior placoid chorioretinitis. Ophthalmology 97:1288-1297

11. Mora P, Borruat FX, Guex-Crosier Y (2005) Indocyanine green angiography anomalies in ocular syphilis. Retina 25:171-181

12. Balaskas K, Sergentanis NT, Giulieri S, Guex-Crosier Y (2011) Analysis of significant factors influencing visual acuity in ocular syphilis. Br J Ophthalmol 96(11):1568-1572

13. Herbort CP, Lehoang P, Guex-Crosier Y (1998) Schematic interpretation of indocyanine green angiography in posterior uveitis using a standard angiographic protocol. Ophthalmology 105:432-440

14. Castellano CG, Stinnett SS, Mettu PS, McCallum RM, Jaffe GJ (2009) Retinal thickening in iridocyclitis. Am J Ophthalmol 148 (3):341-349

15. Wolfensberger TJ, Herbort CP (1999) Indocyanine green angiographic features in ocular sarcoidosis. Ophthalmology 106:285-289

16. Wolfensberger TJ, Piguet B, Herbort CP (1999) Indocyanine green angiographic features in tuberculous chorioretinitis. Am J Ophthalmol 127:350-353

17. Kocak N, Saatci AO, Cingil G, Cimrin A, Ucar ES (2006) Miliary tuberculosis and bilateral multifocal choroidal involvement: place of indocyanine green angiography. Bull Soc Belge Ophtalmol 301:59-65 